Acta Geod. Geoph. Hung., Vol. 43(4), pp. 419-437 (2008)

DOI: 10.1556/AGeod.43.2008.4.4

\title{
PARAMETER SENSITIVITY MAPS OF SURFACE GEOELECTRIC ARRAYS I. LINEAR ARRAYS
}

\author{
S Szalai and L Szarka \\ Geodetic and Geophysical Research Institute of the Hungarian Academy of Sciences, \\ POB 5, H-9401 Sopron, Hungary, e-mail: szalai@ggki.hu; szarka@ggki.hu
}

[Manuscript received January 15, 2008; accepted March 17, 2008]

\begin{abstract}
This paper presents the general features of parameter sensitivity maps and illustrates their practical use. We define parameter sensitivity maps as geoelectric responses in the measuring plane (at the surface) due to an elementary cube within the subsurface at three different depths. Responses of the three component as well as the total response of electrical dipoles formed on opposite cube surfaces are shown. In this paper we present such maps for 14 various linear electrode arrays. This paper is followed by an accompanying one about nonlinear and focussed arrays. Parameter sensitivity maps in these two papers provide a compact characterization of all known geoelectric arrays. We give several examples how to use them in planning and interpretation of field measurements.
\end{abstract}

Keywords: geoelectric arrays; parameter sensitivity; surface geoelectric methods

\section{Introduction}

In the geophysical literature there are altogether about one hundred geoelectric arrays (Szalai and Szarka 2008b). Each of them has specific features, but it is not easy to characterise all these arrays by using a few parameters. The depth of investigation, the vertical resolution are such (one-dimensional) parameters. It is also useful to have an overview about some basic multidimensional features of all those arrays. Responses due to a small elementary resistivity inhomogeneity, put at different $(x, y, z)$ positions within the subsurface, provide such a three-dimensional information. The responses presented in forms of $(x, y)$ planes at various depth levels are called as parameter sensitivity maps.

For the computation of parameter sensitivity values we apply an approach, emerged from the depth of investigation computations by Roy and Apparao (1971). 
In order to determine depth of investigation of various arrays, they needed to compute depth of investigation characteristics (DIC) as a response of a thin sheet within a homogeneous half-space in the subsurface.

Similar computations were carried out by Roy and Dhar (1971) for well logging studies. Parameter sensitivity maps for several surface geoelectric arrays were presented and used for interpretation at first by Barker (1979). The aim of systematic analogue model experiments (a technique, extensively used in the eighties) was in effect providing parameter sensitivity information about various arrays. In the Sopron laboratory anomaly responses ("parameter sensitivity maps") were determined for various dipole-dipole alternating current arrays (Szarka 1994).

Besides the aforementioned first approach, it is possible to compute parameter sensitivity values on basis of the Frechet derivatives (Zhdanov and Keller 1994), namely on the response to an infinitesimal change in the parameters of the multidimensional model. Following this second way, the optimum array (and its optimum parameters) can really be found for the given subsurface model. Among the first geoelectric studies in this field were published by Gyulai (1989) and by Noel and Xu (1991). Noel and Xu (1991) carried out two-dimensional parameter sensitivity studies for geoelectric tomography; Hering et al. (1995) carried out 1D parameter sensitivity studies with the Schlumberger, radial (axial) dipole and two-electrode arrays, in order to reveal their potential in various (geoelectric and seismic-geoelectric) joint inversions; Gyulai (1995) discussed parameter sensitivity of two-electrode, three-electrode, Wenner-, Sclumberger- and radial-dipole arrays in case of inclined layered models; Gyulai (1998) calculated parameter sensitivities in case of several arrays in investigation of subsurface holes. As a practical example of parameter sensitivity studies, Nyári and Kanli (2007) studied effect of small 3D inhomogeneities on 2D interpretation carried out with various arrays. Spitzer and Kümpel (1997) determined the resolution of some three-dimensional model parameters, indicating the contribution of different parts of the model to the overall response.

We think, the two approaches are complementary. This second (the "Frechet derivative" definition is very useful in given field problems, but every problem needs separate and specific numerical studies. At the same time, the first (the "elementary body") approach we follow is suitable to give a general overview about the basic features of the surface geoelectric arrays.

Parameter sensitivity values in forms of maps were carried out by Hursán (1996) and Spitzer (1998) in numerical way. Szalai (1997) computed parameter sensitivity maps by using simple analytical formula, and - as it was demonstrated by Szalai and Szarka (2000) - the analitically computed parameter sensitivity values are in a good agreement with the numerical results, if the subsurface cube is relatively small (the difference is smaller than $5 \%$, if $a / R \leq 0.1$, where $a$ is the side length of the cube, $R$ is the transmitter-receiver distance).

In the first part of the paper we give an insight into the mathematics of our parameter sensitivity calculation, then we summarize the main characteristics of parameter sensitivity maps. Several examples will be also presented to illustrate their practical use. 

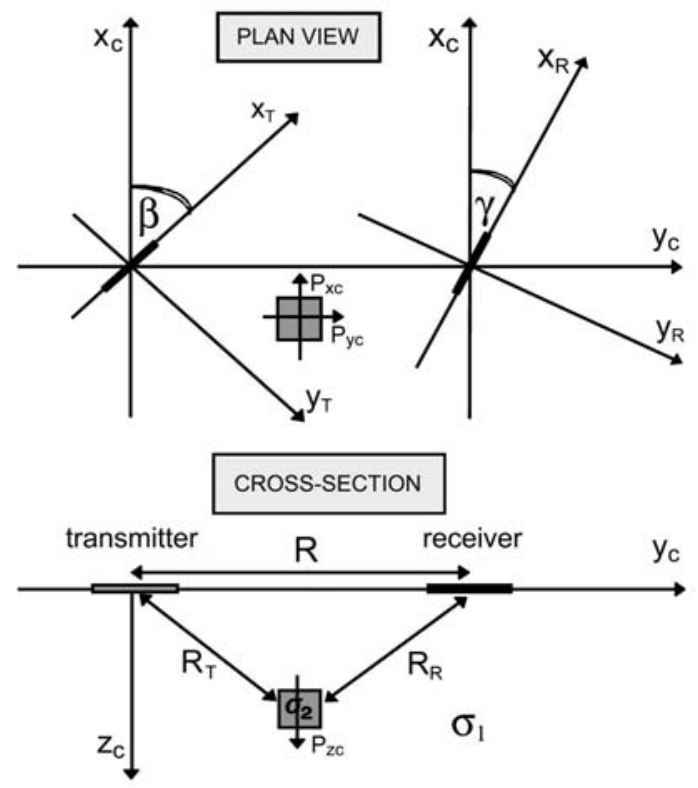

Fig. 1. Notations to compute parameter sensitivity in case of dipole-dipole arrays

In this and in our next, accompanying paper (Szalai and Szarka 2008a, discussing nonlinear and focused arrays) we present a complete list of parameter sensitivity maps of all those geoelectric arrays, which dispose at all parameter sensitivity maps. For this analysis the approximately one hundred arrays, collected by Szalai and Szarka (2008b) were taken into account. In this way, these two papers together characterise all surface geoelectric arrays from a special point of view.

\section{Mathematical derivation}

A cube of side length $a$, of resistivity $\varrho_{1}$ is put in the homogeneous and isotropic half-space of resistivity $\varrho$. (Figure 1 shows a subsurface cube and a dipole-dipole array.) The current flowing into/from the earth through surface electrodes ("cur") is $I$, and the potential difference is measured between surface electrodes $M$ and $N$.

The potential difference $\Delta U^{\text {hom }}$ for a four-electrode system measured over the homogeneous half-space:

$$
\Delta U^{\text {hom }}=\frac{\varrho I}{2 \pi} G^{\text {hom }}, \quad \text { where } \quad G^{\text {hom }}=\sum_{\text {cur }=1}^{n}\left(\frac{1}{r_{\operatorname{cur} M}}-\frac{1}{r_{\operatorname{cur} N}}\right)
$$

where $r_{\operatorname{cur} M}$ and $r_{\operatorname{cur} N}(\operatorname{cur}=1, \ldots, n)$ are the distances of the current electrodes from the $M$ and $N$ electrodes, respectively; $n$ is the number of the current electrodes.

$\Delta U^{\text {total }}$ is the total response in the presence of the cube. $\Delta U^{\text {total }}=\Delta U^{\text {hom }}+$ $\Delta U^{\text {cube }}$, where $\Delta U^{\text {cube }}$ is the net effect of the cube, which is equivalent - from geoelectric point of view — with a dipole moment $\mathbf{p}$. 
Since the surface change density $\tau$ on a resistivity interface $k=\frac{\varrho-\varrho_{1}}{\varrho+\varrho_{1}}$ is $\tau=2 \varepsilon_{0} k E_{b}$ (where $\varepsilon_{0}$ is the dielectric constant, $E_{b}$ is the normal component of the electric field in absence of the interface, as it was shown by Li and Oldenburg 1991), the components $i(i=x, y, z)$ of the dipole moment $\mathbf{p}$, corresponding to the cube, are as follows:

$$
p_{i}=\frac{\varepsilon_{0} \varrho I}{\pi} k a^{3} G_{i}^{\mathrm{cur}}, \quad \text { where } \quad G_{1}^{\mathrm{cur}}=\sum_{\text {cur }=1}^{n} \frac{i_{\mathrm{cur}}-i_{c}}{r_{\mathrm{cur} C}^{3}}
$$

$r_{\text {curC }}($ cur $=1, \ldots, n)$ are the distances of the current electrodes from the centre $C$ of the cube. If

$$
G_{i}^{M N}=\frac{i_{C}-i_{M}}{r_{C M}^{3}}+\frac{i_{N}-i_{C}}{r_{C N}^{3}}, \quad \Delta U^{\mathrm{cube}}\left(p_{i}\right)=\frac{p_{i}}{2 \pi \varepsilon_{0}} G_{i}^{M N}=\frac{\varrho I}{2 \pi^{2}} k a^{3} G_{i}^{\mathrm{cur}} G_{i}^{M N}
$$

The potential difference is the sum of the potential differences due to individual $p_{i}$ dipole components:

$$
\Delta U^{\text {cube }}=\sum U^{\text {cube }}\left(p_{i}\right)=\frac{\varrho I}{2 \pi^{2}} k a^{3} \sum_{i}\left(G_{i}^{\text {cur }} G_{i}^{M N}\right)=\frac{\varrho I}{2 \pi^{2}} k a^{3} G^{\text {cube }} .
$$

The effect of the cube compared to the response due to the homogeneous half-space is

$$
\frac{\Delta U^{\text {cube }}}{\Delta U^{\text {hom }}}=\frac{k a^{3}}{\pi}=\frac{G^{\text {cube }}}{G^{\text {hom }}}
$$

\section{About the parameter sensitivity maps}

Our parameter sensitivity maps show the response due to a small cube in the subsurface, put in any $(x, y, z)$ positions around the current- and potential electrodes. The cube has a side length of $a=0.1 R$, where $R$ is the characteristic array length, i.e. the distance between the two outer electrodes (not at infinity). The values, varying in the horizontal $(x, y)$ plane, show directly the electric potential difference in the given array due to the cube, in the percentage of the corresponding potential difference measured by using a Wenner array over a homogeneous half-space, and normalised by the resistivity contrast.

Parameter sensitivity maps are presented for three different depths: $0.1 R, 0.2 R$ and $0.3 R$.

In this paper we present parameter sensitivity maps for 14 linear arrays. Our maps dispose with some advantageous features as follows.

- Due to normalization procedure (namely, we normalize the parameter sensitivity values with the homogeneous half-space values of the Wenner array), the parameter sensitivity maps of different arrays can be directly compared with each other. In all other publications the normalization is done with the actual maximum, which does not allow any intercomparison. 
- It is possible to show parameter sensitivity maps separately for individual $(x, y$, and $z)$ components. These versions demonstrate, how the accumulated charge at the corresponding cube faces varies as a function of the position of the cube. These maps provide more information than it is shown only in the total cube response.

- We present parameter sensitivity values as well for cube positions not only in the line of the electrodes as it has been wide-spread in the geophysical literature, but also for offline cubes.

- For subsurface bodies of limited size, the values provide good approximation considering even the potential difference values themselves (Szalai and Szarka 2000).

\section{General features of parameter sensitivity maps}

Parameter sensitivity maps - at a given depth of the subsurface body - provide $(x, y)$ distribution of the response values. Generally the investigated area should not be larger than $-0.2 \leq x \leq 1.2$, with characteristic lenght of $R=1,-0.5 \leq y \leq 0.5$.

The positive values indicate an increase in the observed potential difference (or apparent resistivity) due to a high-resistivity subsurface cube, and the negative values indicate a corresponding decrease. The zero isoline separates the two zones. It shows the sites, where the array is practically insensitive to the given subsurface inhomogeneity.

The parameter sensitivity maps can be characterised on basis of their symmetry characteristics, the position of the zero isoline, the relative distribution of the $x, y$ and $z$ sensitivity components, the magnitude of sensitivity values, their areal concentration, and location. This characterization is done later.

\section{Practical advantages of the use of parameter sensitivity maps}

We give examples (1) how to characterize of basic geoelectric array in terms of parameter sensitivity maps, (2) how the basic features of some of new arrays can be understood by using their parameter sensitivity maps, (3) how some new arrays can be constructed on basis of their desired parameter sensitivity maps.

Examples about the basic arrays (1) include:

1a. Understanding the resolution features of electrode arrays (the Schlumberger and the Wenner arrays),

1b. Understanding the effect of inhomogeneities around the current- and potential electrodes (the so-called C-and P effects (Shevnin et al. 1999), or even the static shift problem in magnetotellurics discussed by Spitzer 1998).

Examples about the understanding the mechanism of new arrays (2) will discuss null arrays with the question: can the null arrays promote a better understanding of new arrays and a better preparation of field work? Two examples will be given: 
2a. Null arrays over symmetrical inhomogeneities (Szalai et al. 2000);

2b. Rotational features over elongated inhomogeneities (Szalai et al. 2002).

Examples about construction of new arrays (e.g., Barker 1981) include:

3a. offset-Wenner and

3b. offset square array.

Now we discuss the aforementioned examples as follows.

\section{1a. Resolution of Schlumberger and Wenner arrays}

Comparing the Schlumberger and the Wenner arrays, it is evident that in case the sensitivity distribution of the Schlumberger array (Fig. 5) is much more concentrated than that of the Wenner array (Fig. 6), which has much smoother parameter sensitivity maps. This is the explanation for the superiority of Schlumberger array over the Wenner array in terms of resolution. The large, extended anomaly zones are indications of the robustness of the array, since the information content varies only slowly along the profile. It means that the Wenner array is very robust. In noisy areas robust arrays (e.g., Wenner) should be preferred, but in less noisy areas, especially when high resolution is needed, non-robust arrays (e.g., Schlumberger) represent a good choice.

\section{1b. $C$ and $P$ effects in VES (Shevnin et al. 1999)}

The $\mathrm{C}$ and $\mathrm{P}$ effects (Shevnin et al. 1999) can be understood by using the parameter sensitivity map of the Schlumberger array. Since the environment of the $\mathrm{P}$ electrodes does not change in the sounding process (until the next increase of electrode distance, in order to increase the lower and lower potential difference), it produces only a parallel shift (the so-called $\mathrm{P}$ effect) on the sounding curves. At the same time, the environment of the moving current electrodes is changing in each step. This may cause a characteristic distortion of the sounding curves, the so-called "double-anomalies". (See the opposing signs at the two sides of the same current electrode.)

\section{2a. Null-arrays over symmetric inhomogeneities (Szalai et al. 2002)}

The null arrays in general have antisymmetry features (e.g. Fig. 18). The Schlumberger null-array (Szalai et al. 2002) has two antisymmetry axes (Fig. 2 a): the $y=0$ and the $x=0.5$ lines. Due to this fact, any resistivity inhomogeneity (either 2D or 3D), having a symmetry axis at either $y=0$ or $x=0.5$, is simply not detected by using this array! With other words: the effect of the symmetrical inhomogeneity element pairs just eliminate each other. 

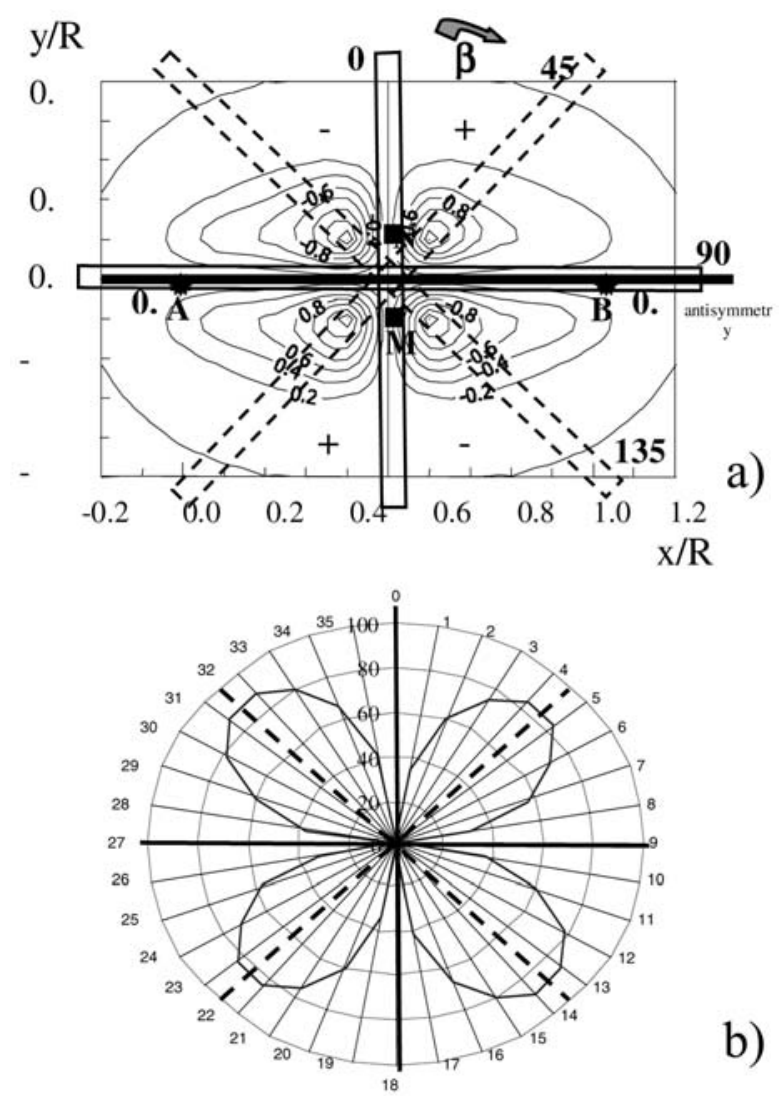

b)

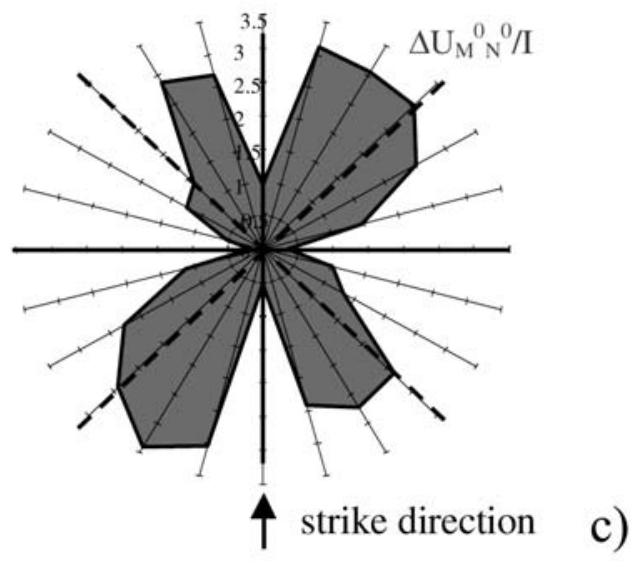

Fig. 2. a) Parameter sensitivity map of the Schlumberger null array, and the surface projections of the highly conductive layer. b) Polar diagram from physical model experiment over a graphite sheet. c) Polar diagram measured in the field above a fissure filled by clay. In b and c the absolute values of potential differences are shown 

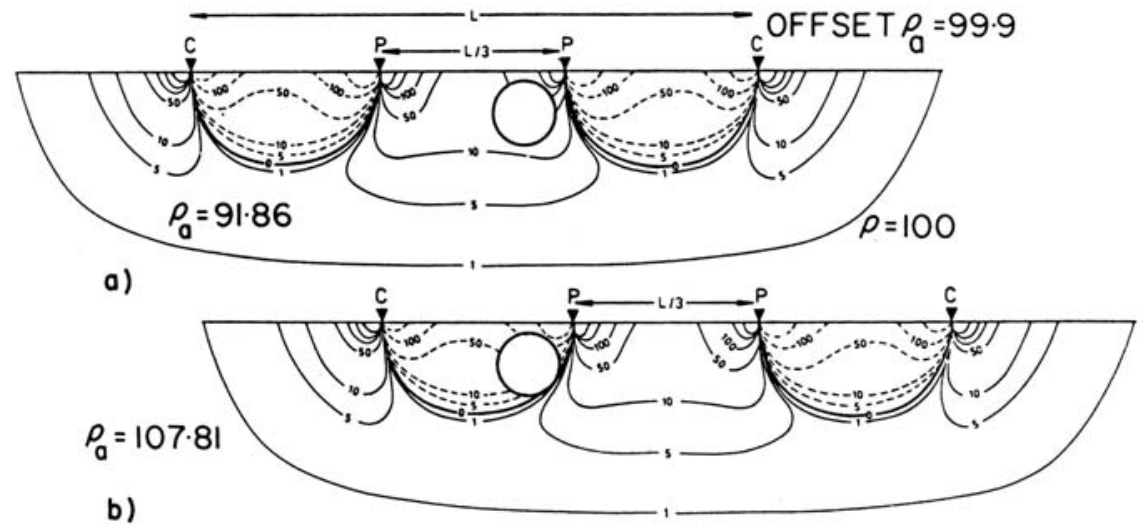

Fig. 3. a) Cross section of the signal composition of the Wenner array with a high conductivity sphere (negative values), in case of a background resistivity of $100 \mathrm{ohmm}$. b) Offset position of the electrodes. The sphere is now in the negative tone. Distortion of isolines due to the presence of the sphere is not shown (after Barker 1981)

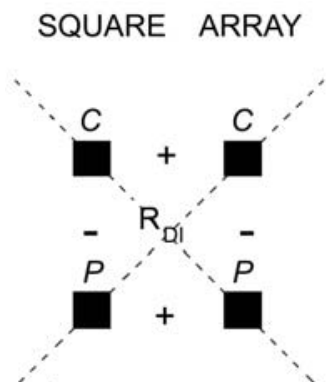

a) ALPHA

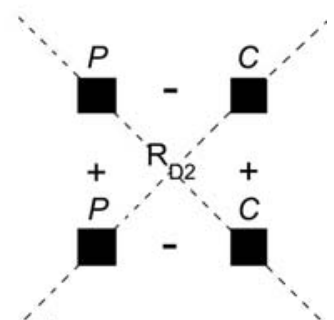

b) GAMMA

$$
\mathrm{R}_{\mathrm{D}}=\left(\mathrm{R}_{\mathrm{D} 1}+\mathrm{R}_{\mathrm{D} 2}\right) / 2
$$

Fig. 4. Parameter sensitivity signs of the a) square- $\alpha$ and b) square- $\gamma$ arrays. The contribution of the near-surface zone between the electrodes at first is positive, then it is negative (after Barker 1981)

\section{2b. Rotational studies over conductivity inhomogenities (Szalai et al. 2002)}

By means of parameter sensitivity maps it is possible to construct a method to determine the direction of elongated conductivity inhomogeneities. Namely, by rotating the so-called Schlumberger null array over the inhomogeneity, it is evident from $2 \mathrm{a}$ that the parameter sensitivity is zero when the array is parallel to the inhomogeneity and when it is perpendicular to it. In other situations the response is nonzero (Fig. 2 a). Consequently, the characteristic directions of the inhomogeneity can be determined from the rose diagram of the observed values (Szalai et al. 2002, and results of an analogue modelling experiment (Fig. 2 b) and a field measurement (Fig. 2 c). 

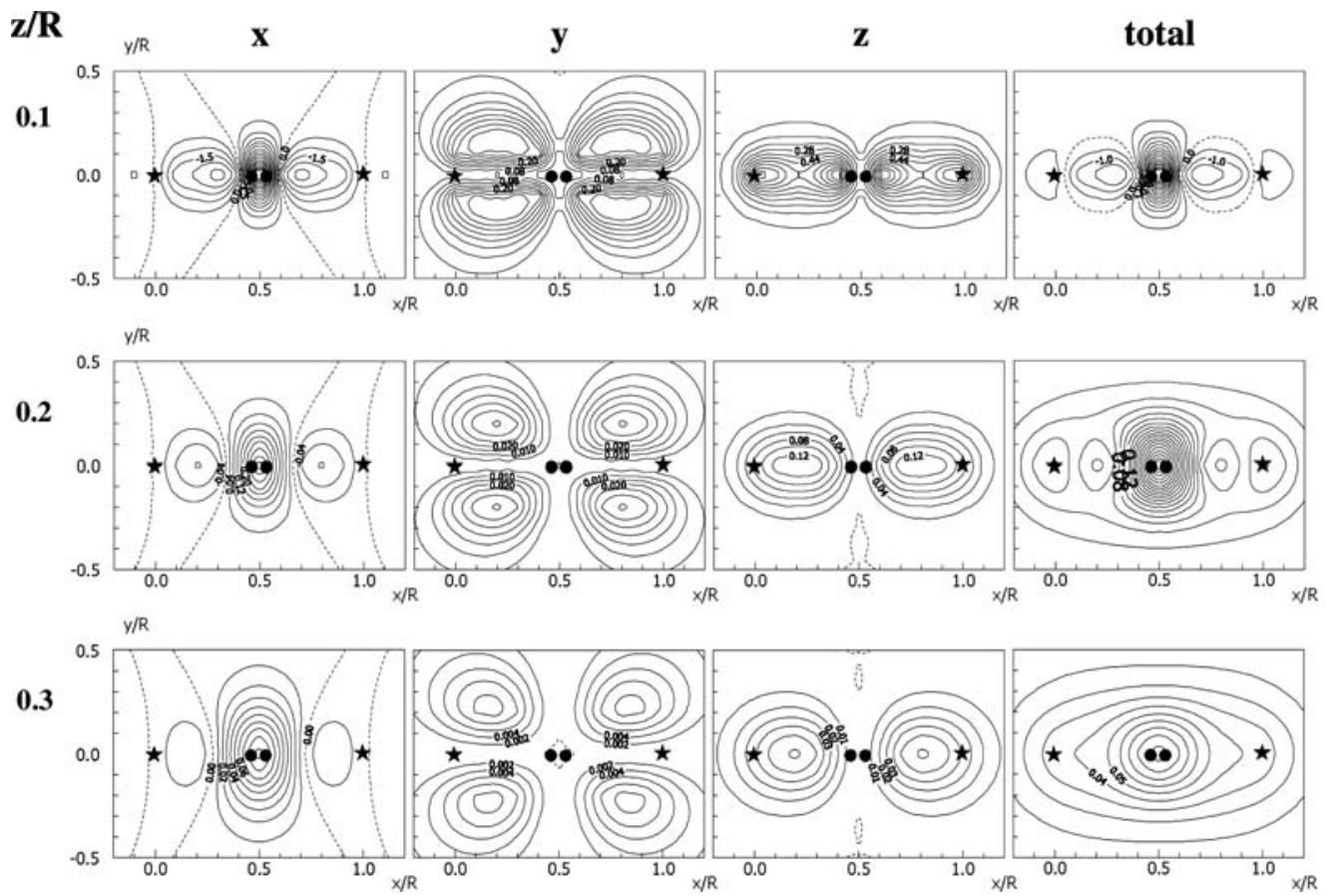

Fig. 5. Parameter-sensitivity map series of the Schlumberger array (No. 1 in Table I), with the current electrode (stars) and potential electrode (circles) positions. $x, y, z$ components illustrate the effect of electrical charges accumulated at the corresponding opposite cube faces, while "total" means their superposed effect. The maps were made at three various depths/characteristic length values $(0.1,0.2$ and 0.3$)$

\section{3a. Offset-Wenner array (Barker 1981)}

In case of the Wenner array (Fig. 6), the dominating electric dipol is $x$-directed, and the effect is the most significant in central position. Deep bodies appear only with positive sign, but near-surface small bodies appear either with positive or with negative sign, depending on their $x, y$ position. Barker (1981) used this feature for the construction of his offset Wenner array as follows: the same near-surface inhomogeneity by using two Wenner arrays (when the second one is shifted by the distance between the current electrodes), is detected with the same sensitivity values, but with opposite signs (Fig. 3). Consequently the arithmetic mean value of the two measurements eliminates near-surface effects, and the resulting mean values are more appropriate for soundings.

The $y$-directed dipoles have influence only in off-side position; the z-directed dipoles only in the two outer $R / 3$ sections. 

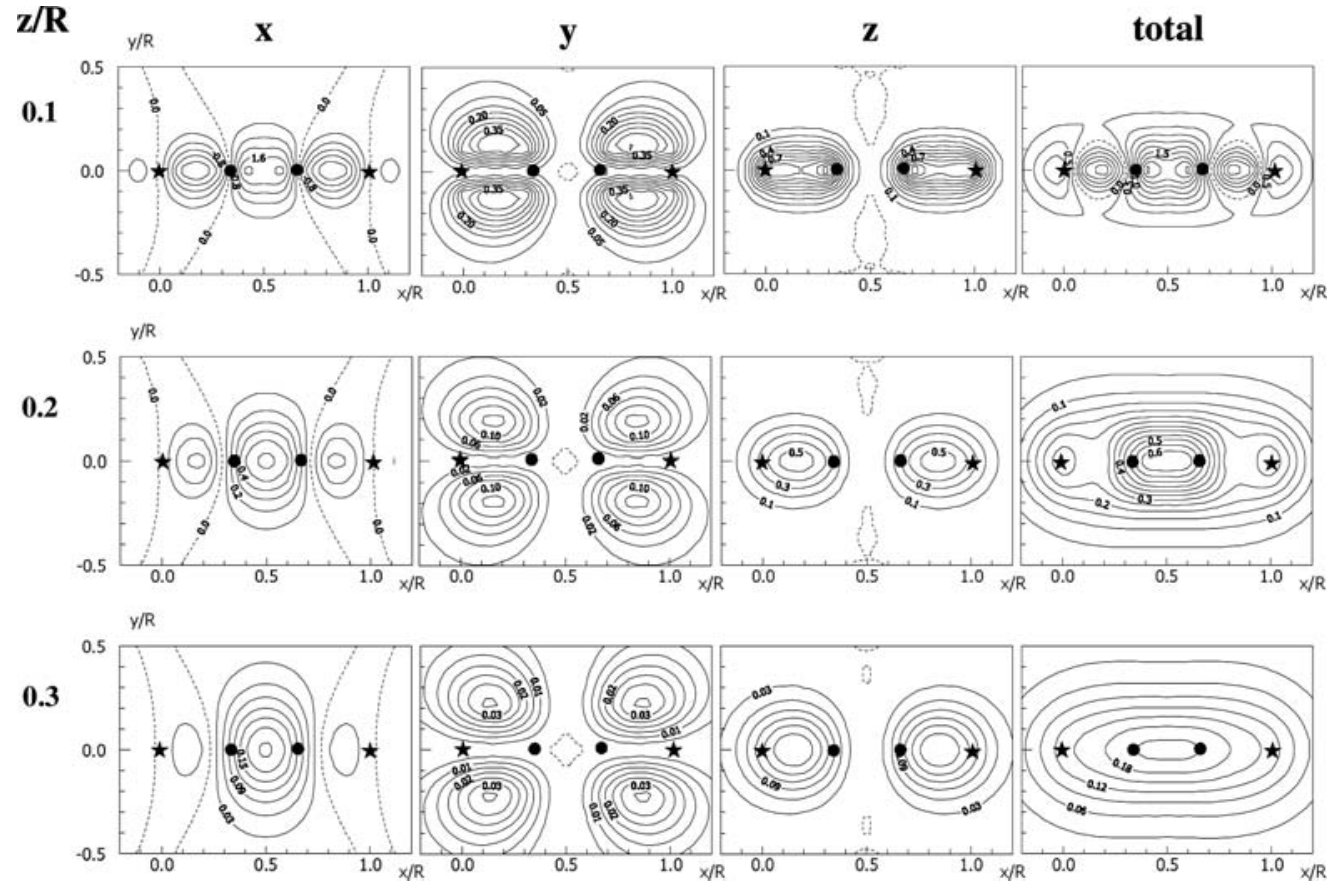

Fig. 6. Parameter-sensitivity map series of the Wenner array (No. 2 in Table I). For notations see Fig. 5

\section{3b. Offset square array (Barker 1981)}

In case of the square- $\alpha$ array for the $y$ and $z$ dipoles the high sensitivity zones are those between the closest potential electrode - current electrode pairs, while the $x$ dipole has a higher sensitivity in the central zone (Fig. 4, showing only the sign of parameter sensitivity values in the four quarters). When the so-called square$\alpha$ array is rotated by 90 degrees, and when a mean value is taken from the two measurements, we arrive to the offset-square array (Barker 1981).

\section{Parameter sensitivity maps of linear electrode arrays}

In the following we present parameter sensitivity maps of 14 linear arrays (Figs 5-18). The corresponding electrode co-ordinates are shown in Table I.

The parameter sensitivity maps of symmetrical arrays are also symmetrical.

Array a0105 (Fig. 7), a0304 (Fig. 8), ght (Fig. 9), half-Wenner (Fig. 10), halfSchlumberger (Fig. 11), quasi-MAN (Fig. 17), and MAN (Fig. 18) arrays have one symmetry axis (in the line of the electrodes). At the same time, the Schlumberger (Fig. 5), the Wenner (Fig. 6), the two-electrode (Fig. 12), the Wenner- $\beta$ (Fig. 13), the dipole-axial (Fig. 14), the Wenner- $\gamma$ (Fig. 15), and the Twin (Fig. 16) arrays have two symmetry axes. Among the aforementioned arrays it is only the MAN array (Fig. 18) which has antisymmetry axis, perpendicular to the connecting line of the electrodes. If an array has antisymmetry axis, it has zero potential difference 
Table I. Electrode co-ordinates of the fourteen linear arrays for the computation of the parameter sensitivity maps shown in this paper

\begin{tabular}{|c|c|c|c|c|c|c|c|c|c|c|c|}
\hline \multirow{2}{*}{$\begin{array}{l}\text { Number of } \\
\text { electrodes }\end{array}$} & \multirow{2}{*}{$\begin{array}{l}\text { Type of } \\
\text { the array }\end{array}$} & \multirow{2}{*}{$\begin{array}{l}\text { Number of } \\
\text { the array }\end{array}$} & \multirow{2}{*}{$\begin{array}{l}\text { Name of } \\
\text { the array }\end{array}$} & \multicolumn{2}{|c|}{$\mathrm{A}$} & \multicolumn{2}{|l|}{ B } & \multicolumn{2}{|c|}{ M } & \multicolumn{2}{|c|}{$\mathrm{N}$} \\
\hline & & & & $\mathrm{x}$ & $\mathrm{y}$ & $\mathrm{x}$ & $\mathrm{y}$ & $\mathrm{x}$ & $\mathrm{y}$ & $\mathrm{x}$ & $\mathrm{y}$ \\
\hline & & 1 & Schlumberger & 0 & 0 & 1 & 0 & 0.45 & 0 & 0.55 & 0 \\
\hline & & 2 & Wenner- $\alpha$ & 0 & 0 & 1 & 0 & 0.33 & 0 & 0.67 & 0 \\
\hline & & 3 & $\mathrm{a} 0105$ & 0 & 0 & 1 & 0 & 0.1 & 0 & 0.5 & 0 \\
\hline & & 4 & a0304 & 0 & 0 & 1 & 0 & 0.3 & 0 & 0.4 & 0 \\
\hline & & 5 & ght & 0 & 0 & 1 & 0 & 0.1 & 0 & $\inf$ & 0 \\
\hline & & 6 & Half-Wenner & 0 & 0 & $\inf$ & 0 & 0.5 & 0 & 1 & 0 \\
\hline Four & Linear & 7 & Half-Schlumberger & 0 & 0 & $\inf$ & 0 & 0.9 & 0 & 1 & 0 \\
\hline electrodes & array & 8 & Two-electrode & 0 & 0 & $\inf$ & 0 & 1 & 0 & $\inf$ & 0 \\
\hline & & 9 & Wenner- $\beta$ & 0 & 0 & 0.33 & 0 & 0.67 & 0 & 1 & 0 \\
\hline & & 10 & Dipole axial & 0 & 0 & 0.1 & 0 & 0.9 & 0 & 1 & 0 \\
\hline & & 11 & Wenner- $\gamma$ & 0 & 0 & 0.67 & 0 & 0.33 & 0 & 1 & 0 \\
\hline & & 12 & Twin & 0 & 0 & 0.9 & 0 & 0.1 & 0 & 1 & 0 \\
\hline & & 13 & Quasi-MAN & 0 & 0 & 0.9 & 0 & 0.8 & 0 & 1 & 0 \\
\hline & & 14 & MAN & 0 & 0 & 1 & 0 & 0.5 & 0 & $\inf$ & 0 \\
\hline
\end{tabular}

in all symmetrical positions over three- and two-dimensional inhomogeneities, and also over any one-dimensional situation.

The $(x, y)$ distribution of sensitivities is very concentrated in case of all arrays, where two electrodes are close to each other: either the two potential electrodes (Schlumberger array in Fig. 5, half-Schlumberger in Fig. 11, dipole-axial array in Fig. 14) or one of the potential electrodes and one of the current electrodes (a0105 in Fig. 7, ght Fig. 9, Twin, Fig. 16, gtt, Fig. 17). These arrays dispose with good resolution. The Wenner-related arrays (Wenner- $\alpha$ in Fig. 6, Wenner- $\beta$ in Fig. 13, Wenner- $\gamma$ in Fig. 15, and half-Wenner in Fig. 10) have much lower but more reliable values. (They are less sensitive to small near-surface disturbing bodies.)

Parameter sensitivity of some arrays vary strongly on depth (e.g., dipole axial array in Fig. 14), while of some other array vary less strongly (e.g., the MAN array in Fig. 18).

It is a general rule that toward larger depths the elementary anomalies are more and more blurred.

In case of each linear array the lateral conductivity variations, parallel to the array, are the most decising factor; although at larger and larger depths the vertical changes become relatively more and more significant. Parameter sensitivity maps of the so-called "half" arrays (e.g., half-Schlumberger or half-Wenner arrays in Fig. 11 and in Fig. 10) are very similar to the half of the parameter sensitivity maps of the original "full" array.

It is worth discussing the sites where small-size inhomogeneities cannot be observed. They are shown with dashed lines in the parameter sensitivity maps. Their geometry may be linear (e.g., the $x$ component of the Wenner array, see Fig. 5), circular (e.g., the total sensitivity in case of the same array, as seen in Fig. 5). In case of some arrays such null zones do not exist, and all sensitivity values have the 
$\mathbf{z} / \mathbf{R}$
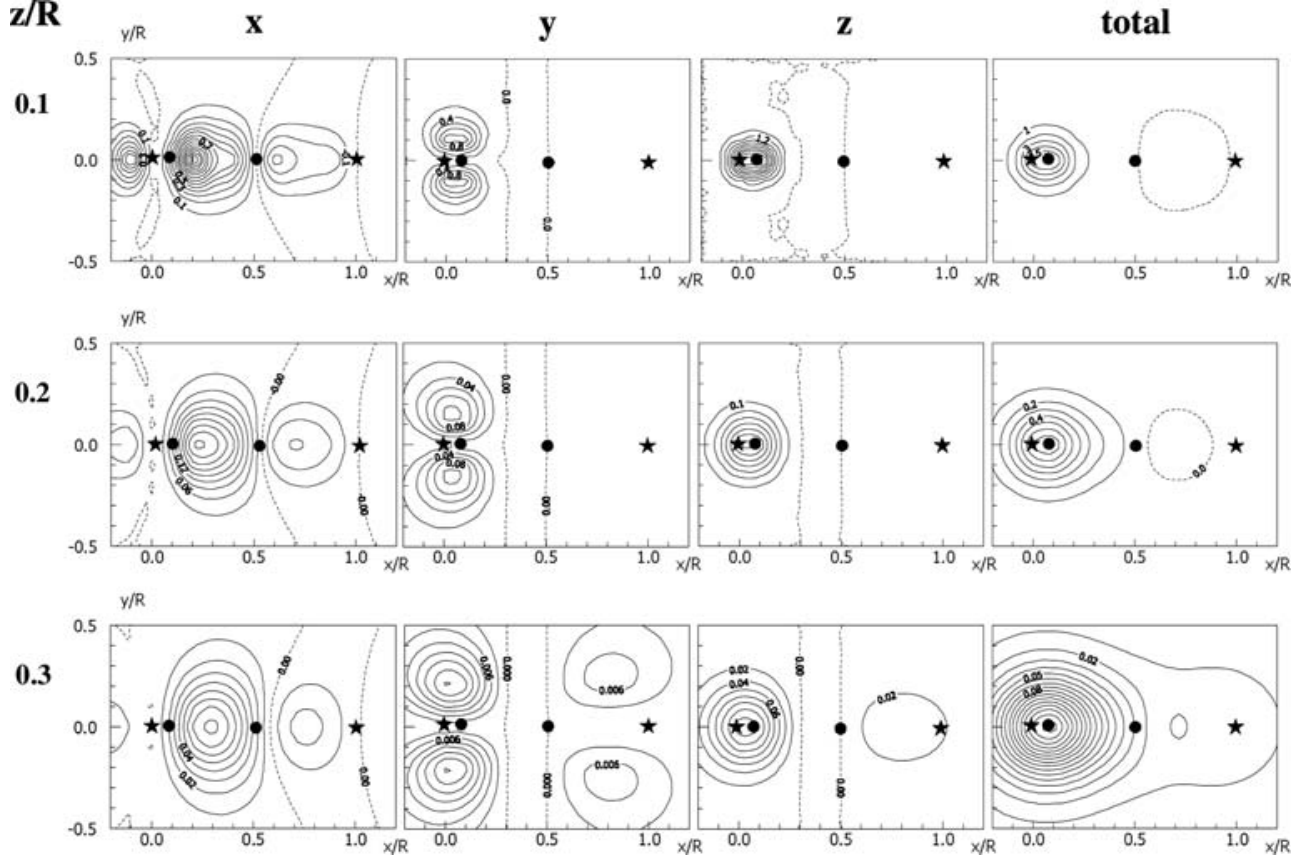

Fig. 7. Parameter-sensitivity map series of the a0105 array (No. 3 in Table I). For notations see Fig. 5
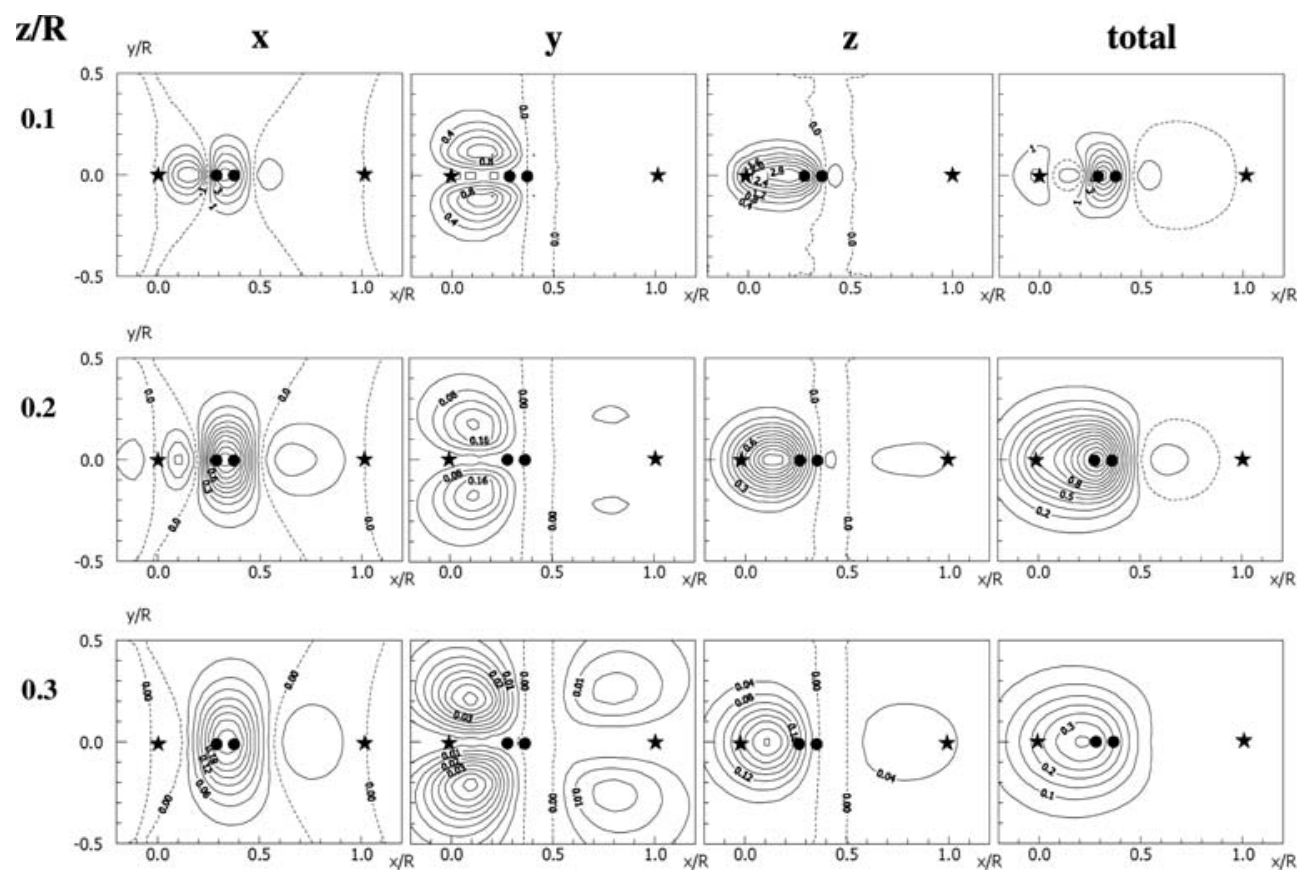

Fig. 8. Parameter-sensitivity map series of the a0304 array (No. 4 in Table I). For notations see Fig. 5

Acta Geod. Geoph. Hung. 43, 2008 

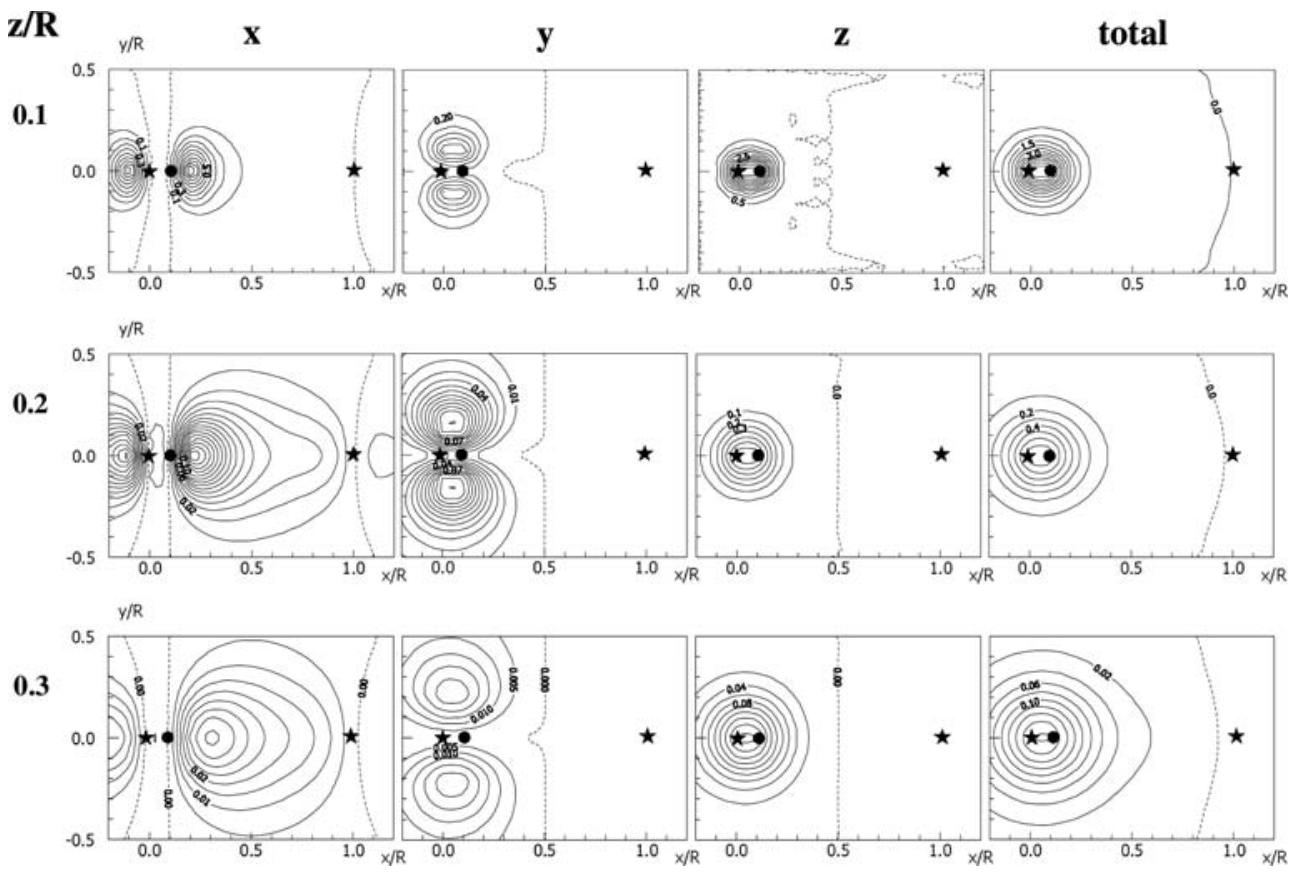

Fig. 9. Parameter-sensitivity map series of the ght ( $\gamma$-type half-Twin) array (No. 5 in Table I). For notations see Fig. 5
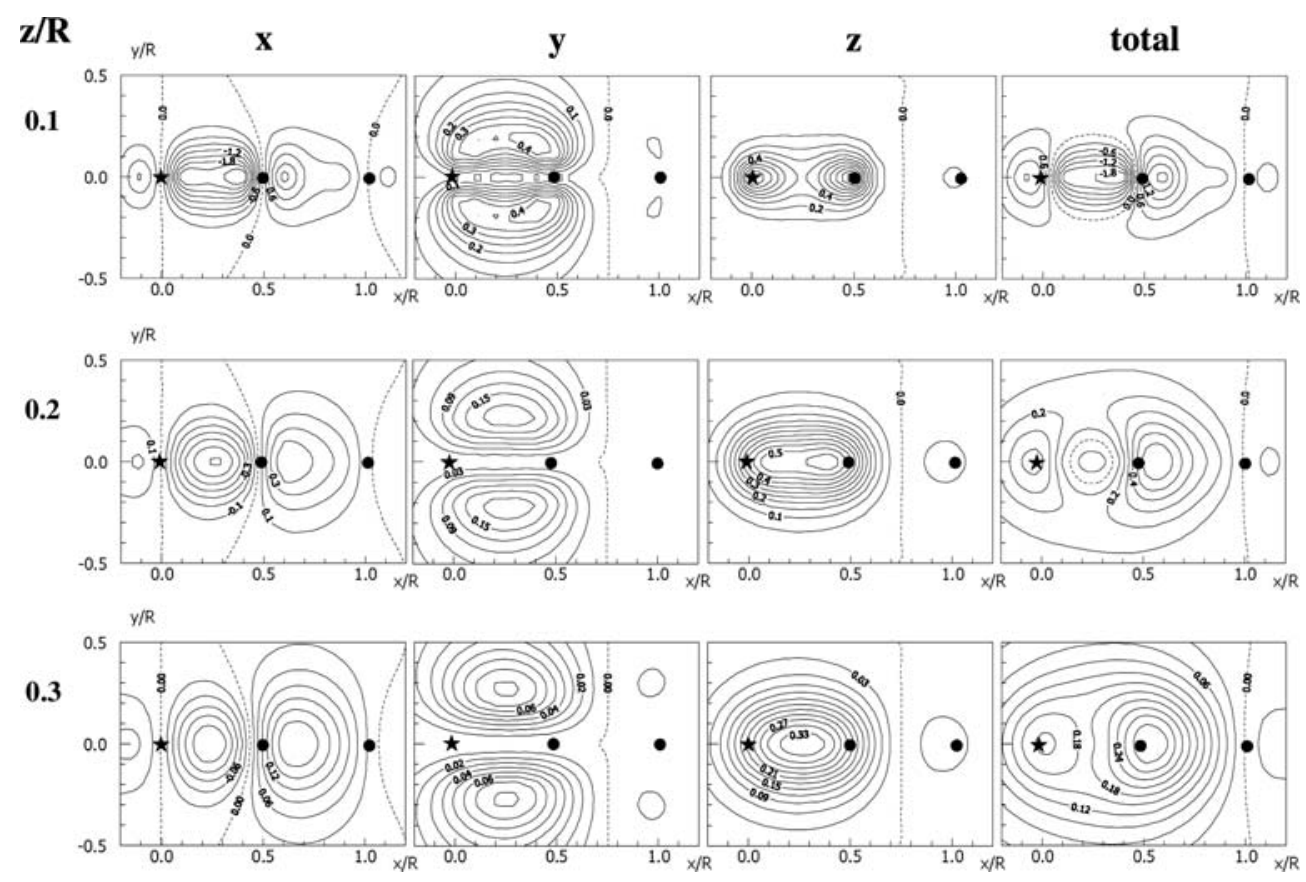

Fig. 10. Parameter-sensitivity map series of the half-Wenner (pole-dipole) array (No. 6 in Table I). For notations see Fig. 5 
$\mathbf{z} / \mathbf{R}$
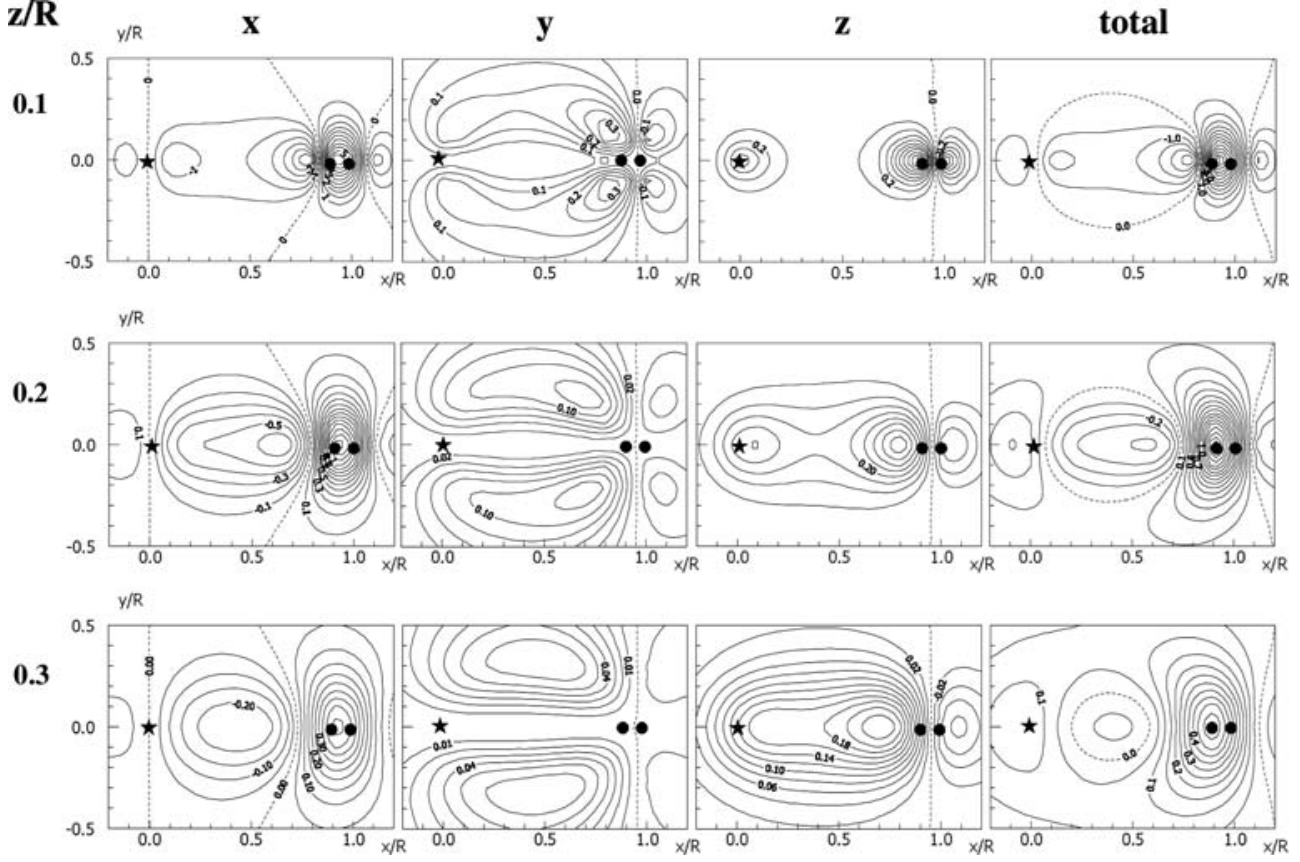

Fig. 11. Parameter-sensitivity map series of the half-Schlumberger (three-electrode) array (No. 7 in Table I). For notations see Fig. 5
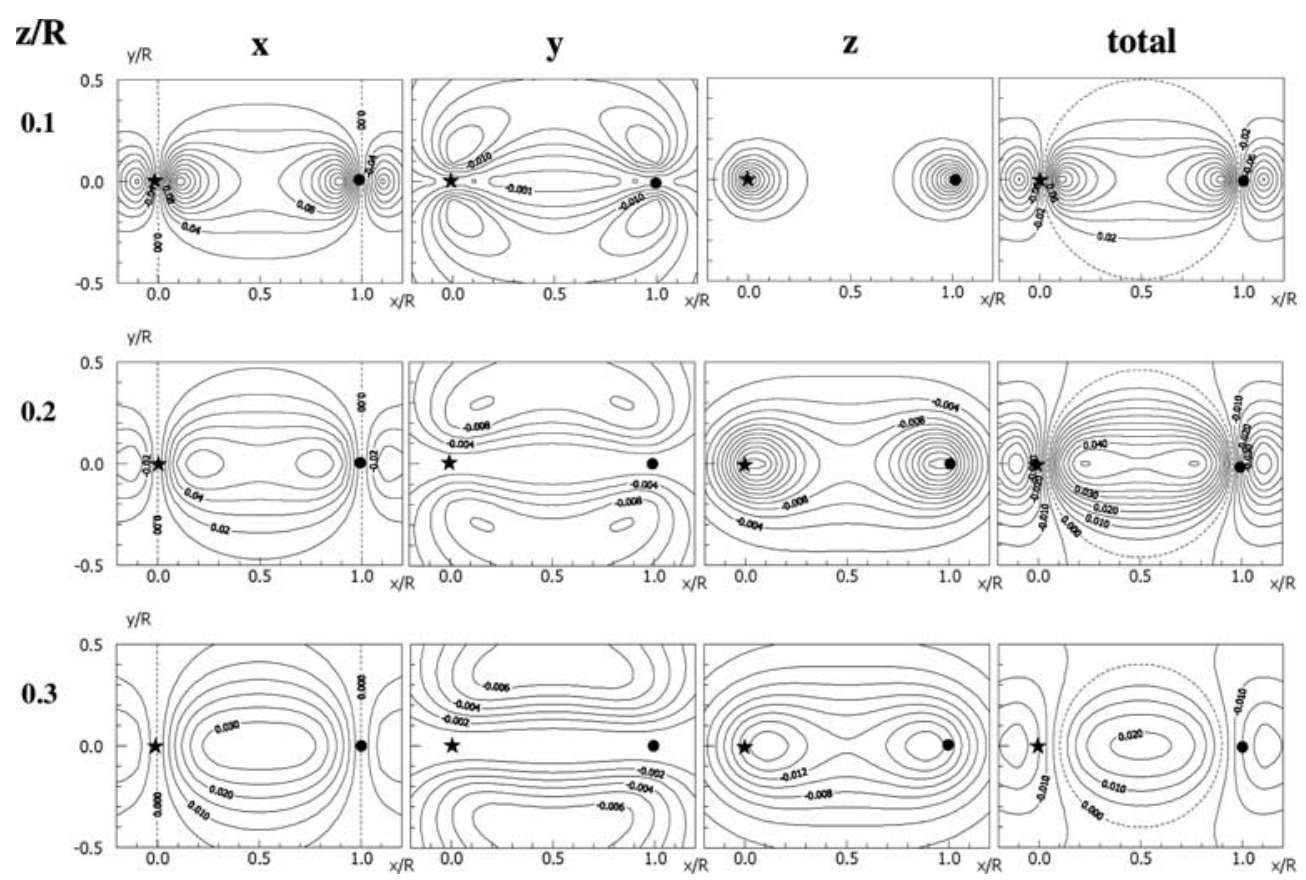

Fig. 12. Parameter-sensitivity map series of the two-electrode array (No. 8 in Table I). For notations see Fig. 5

Acta Geod. Geoph. Hung. 43, 2008 
same sign (e.g. $y$ component of the Wenner array, Fig. 5). In general, the main sensitivity zone of the linear arrays are beneath the array line.

By means of parameter sensitivity maps the effect of current focussing can also be studied. One example: In case of the Wenner array (Fig. 6), increasing the depth from $z / R=0.1$ to $z / R=0.3$, the increase of the $z$ component of the parameter sensitivity value is about 2 (from appr. 0.5 to appr. $\sim 0.9$ ). At the same time, in case of the unipole Wenner- $\alpha$ array, this increase is about 10 (from 0.6 to about 6 ). It means that the $z$ component becomes much more dominant in case of the focussed array than it was in case of the non-focussed one. Studying the $z$ component sensitivity is important e.g., in the delineation of an oil layer floating on groundwater.

It is not the object of this paper to exploit all potentials of parameter sensitivity maps. We have just given examples how to use them.

\section{Conclusions}

We have presented parameter sensitivity maps of various linear geoelectric arrays. Our parameter sensitivity maps are results of simple analytical formula, and its advantages are as follows:

1. the parameter sensitivity values obtained for different arrays can be directly compared to each other,

2 . the components of the physical source of anomaly (the dipoles of electrical charges at opposite cube faces) can be also studied.

The large, extended anomaly zones are indications of the robustness of the array since the information content varies only slowly along the profile. For example the Wenner array is very robust. Arrays, disposing small-size anomalies in the parameter sensitivity (e.g. in the case of the Schlumberger array), are more sensitive to lateral variations. In noisy areas robust arrays should be preferred, but in less noisy areas, especially when high resolution is needed, non-robust arrays are a good choice.

The depth variation of parameter sensitivity informs about the depth of investigation. Integrating the parameter sensitivity values in the horizontal plane, normalised 1D depth of investigation values can be calculated. Keeping the depth variation in mind, laborious numerical and/or physical modelling experiments can be significantly reduced.

By means of parameter sensitivity maps e.g., the effect of current focussing can also be studied.

The presented set of parameter sensitivity maps are open to any further analysis.

Our parameter sensitivity study helps in understanding the physics of the resulting anomalies and basic features of various arrays. We recommend their methodological use in geoelectric prospecting. For example this presentation opens the way to construct new arrays. However, it is not guaranteed that an array having favourable parameter sensitivity maps over the elementary body, will certainly have equally favourable properties in investigation of some large-size multidimensional bodies. 
$\mathbf{z} / \mathbf{R}$ $\mathbf{x} \quad \mathbf{y} \quad \mathbf{z}$

$\mathbf{z} \quad$ total

0.1
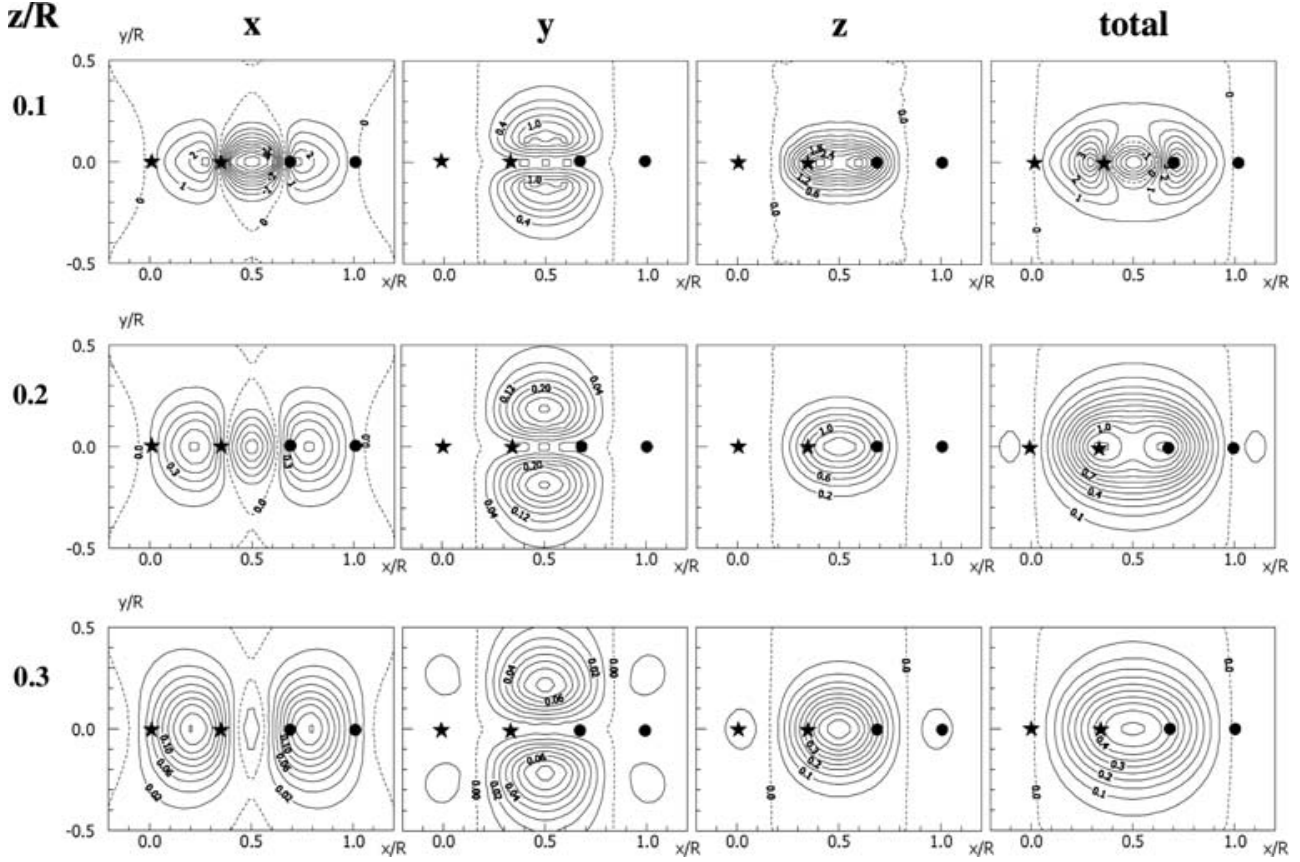

Fig. 13. Parameter-sensitivity map series of the Wenner- $\beta$ array (No. 9 in Table I). For notations see Fig. 5
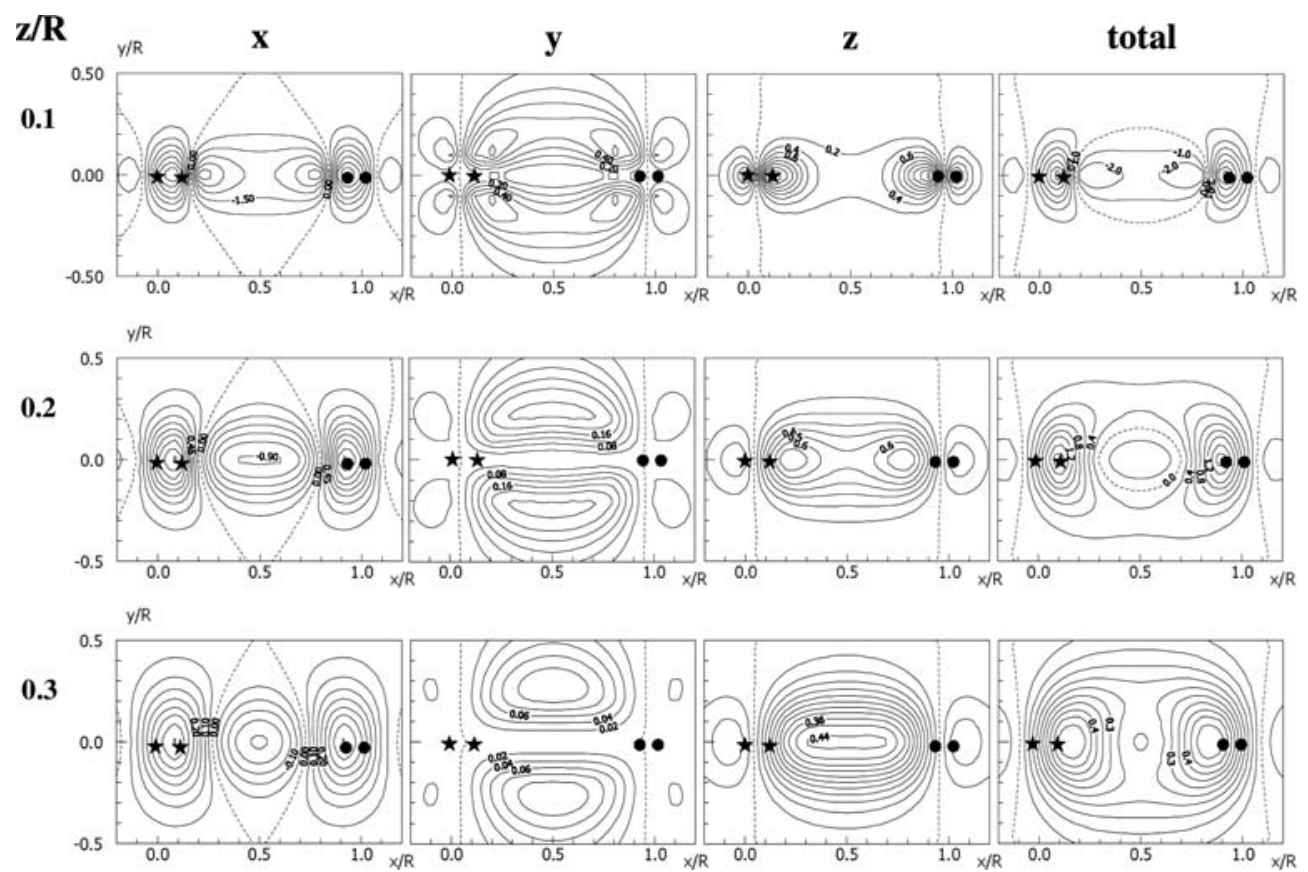

Fig. 14. Parameter-sensitivity map series of the dipole-axial array (No. 10 in Table I). For notations see Fig. 5

Acta Geod. Geoph. Hung. 43, 2008 
$\mathbf{z} / \mathbf{R}$
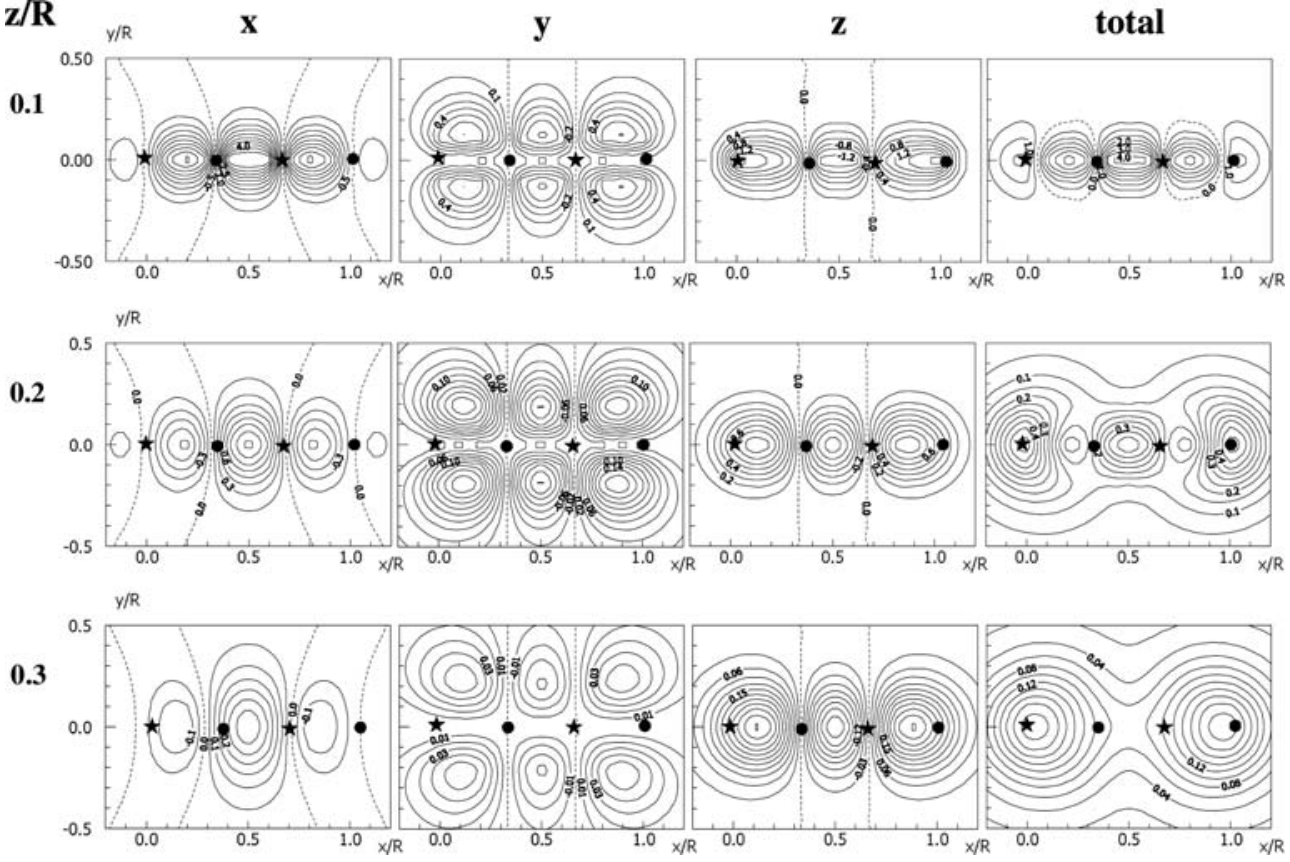

Fig. 15. Parameter-sensitivity map series of the Wenner- $\gamma$ array (No. 11 in Table I). For notations see Fig. 5
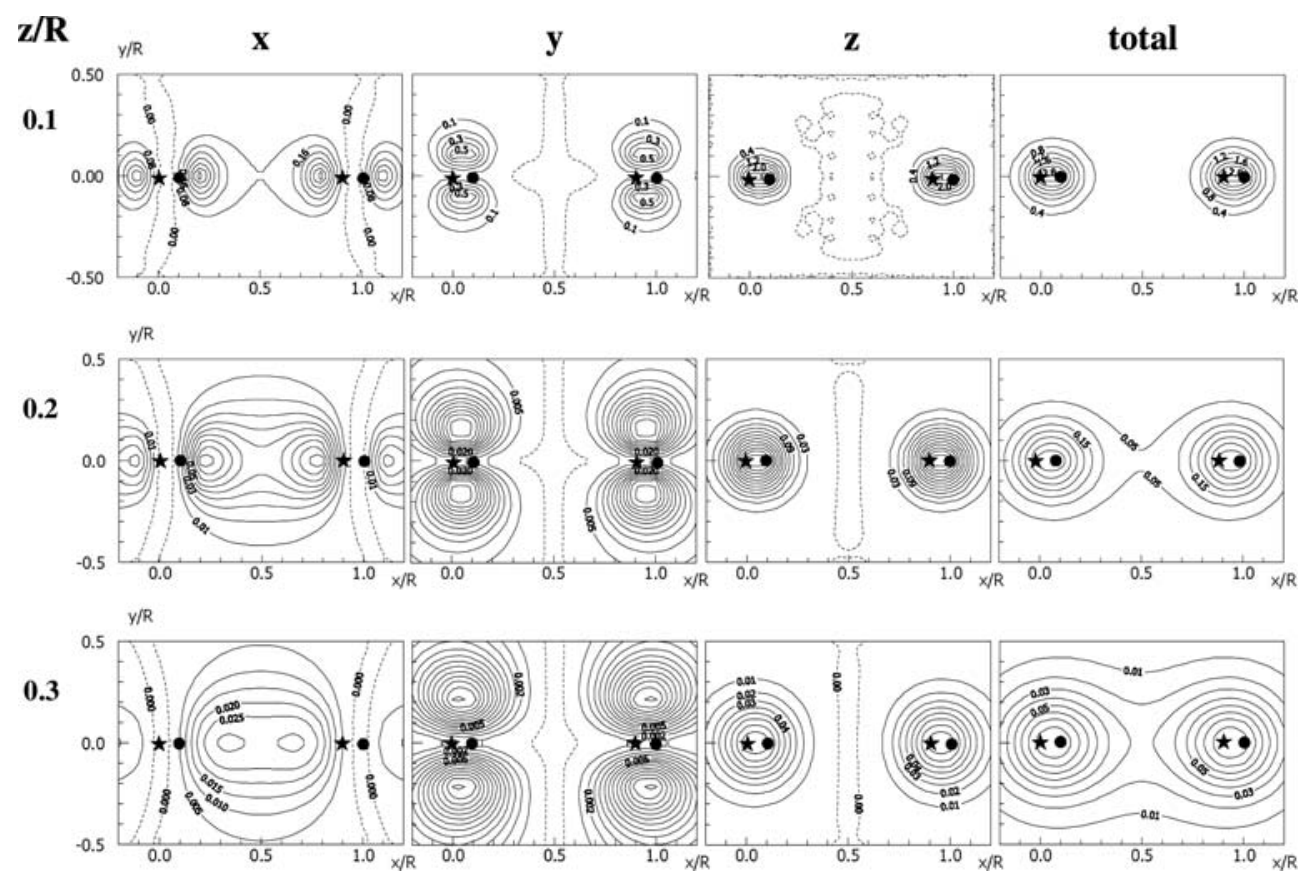

Fig. 16. Parameter-sensitivity map series of the Twin array (No. 12 in Table I). For notations see Fig. 5 
$\mathbf{z} / \mathbf{R}$

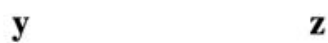

$\mathbf{Z}$

total

0.1
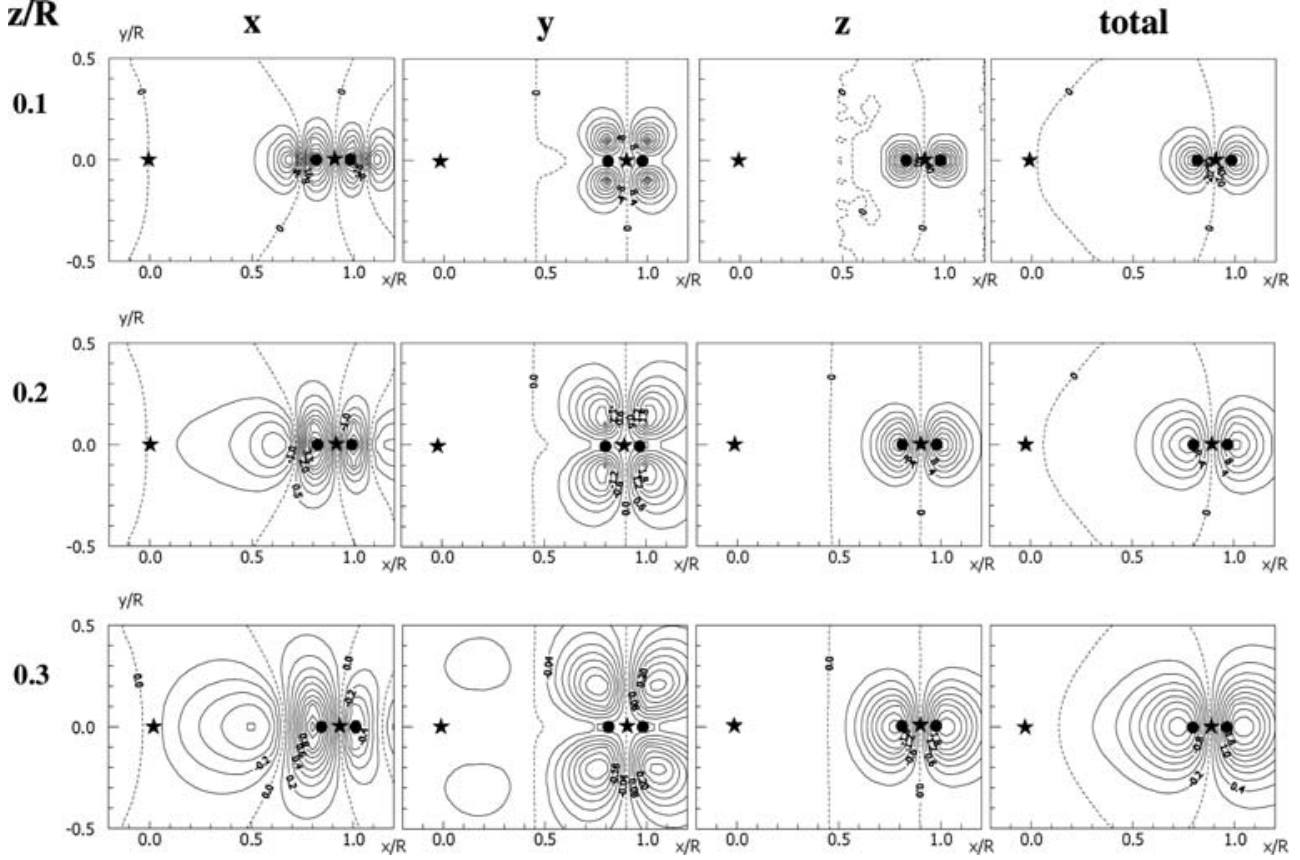

Fig. 17. Parameter-sensitivity map series of the quasi-MAN array (No. 13 in Table I). For notations see Fig. 5
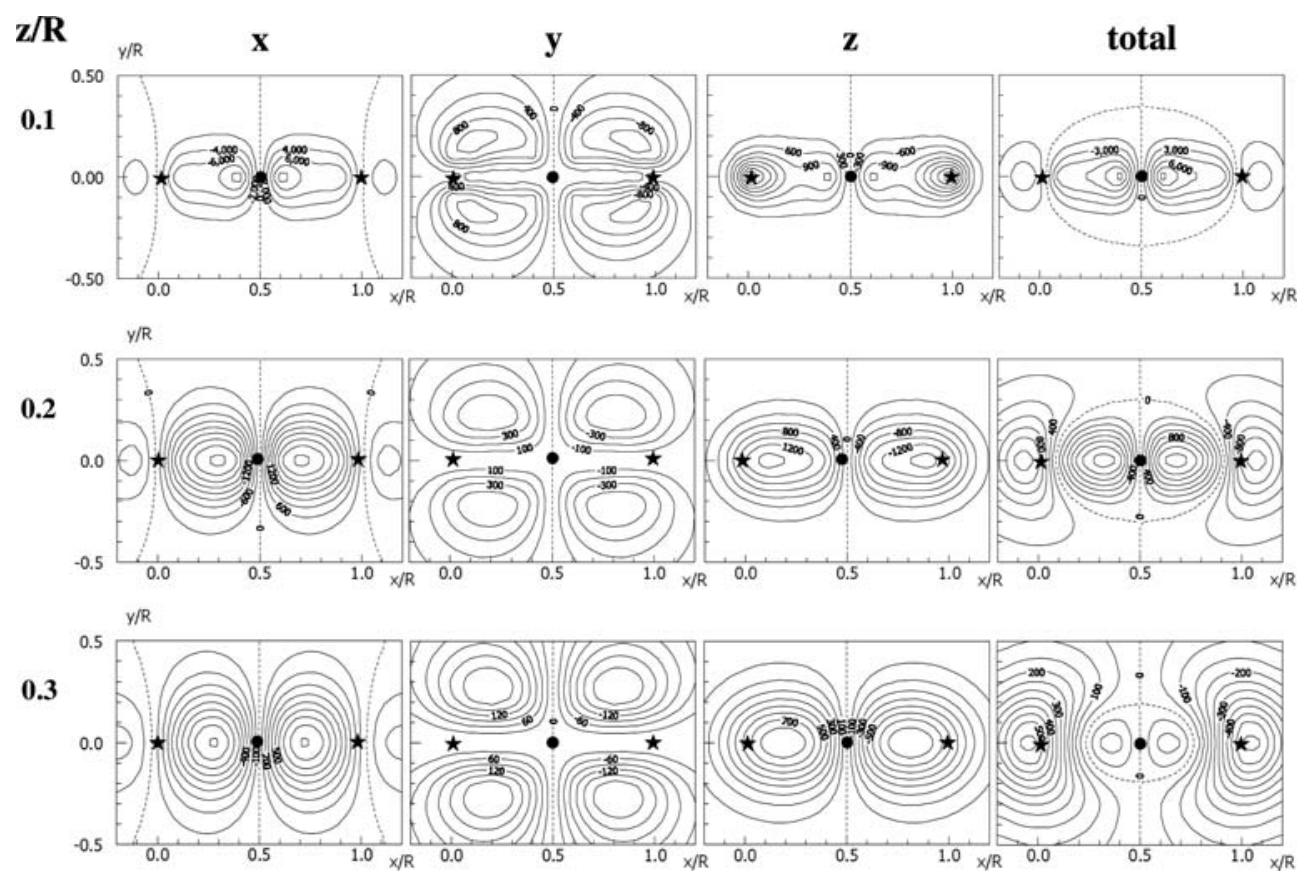

Fig. 18. Parameter-sensitivity map series of the MAN (midpoint-null) array (No. 14 in Table I). For notations see Fig. 5

Acta Geod. Geoph. Hung. 43, 2008 


\section{Acknowledgements}

Hungarian National Scientific Research Fund (projects K49604 and NI 61013); Bolyai János Research Scholarship of the Hungarian Academy of Sciences (Sándor Szalai); Ákos Gyulai and an anonymous referee (for their valuable comments).

\section{References}

Barker R D 1979: Geophys. J. R. astr. Soc., 39. 123-129.

Barker R D 1981: Geophys. Prosp., 29, 128-143.

Gyulai Á 1989: Geophys. Trans., 35, 209-225.

Gyulai Á 1995: Magyar Geofizika (in Hungarian), 36, No. 1, 40-67.

Gyulai Á 1998: Magyar Geofizika (in Hungarian), 39, No. 2, 43-50.

Hering A, Misiek R, Gyulai Á, Ormos T, Dobróka M, Dresen L 1995: Geophys. Prosp., 43, No. 2, 135-156.

Hursán G 1996: Magyar Geofizika (in Hungarian), 37, No. 2, 106-117.

Li Y, Oldenburg D W 1991: Geophys. Prosp., 39, 803-826.

Noel M, Xu B 1991: Geophys. J. Int., 107, 95-102.

Nyári Zs, Kanli A I 2007: J. Geophys. Eng., 4, 83-93.

Roy A, Apparao A 1971: Geophysics, 36, 943-959.

Roy A, Dhar R L 1971: Geophysics, 36, 754-760.

Shevnin V A, Bobachev A A, Modin I N, Pervago E V 1999: Interpretation of resistivity sounding data - distorted by geological noise. 61st EAGE Meeting 1999. P027

Spitzer K 1998: Geophys. J. Int., 134, 734-746.

Spitzer K, Kümpel H J 1997: Geophys. Prosp., 45, 963-982

Szalai S 1997: 3D parameter-sensitivity of d.c. dipole arrays. EAGE 59th Meeting 1997. P136

Szalai S, Szarka L 2000: Geophys. Prosp., 48, 871-885.

Szalai S, Szarka L, Prácser E, Bosch F, Müller I, Turberg P 2002: Geophysics, 67, 1769-1778.

Szalai S, Szarka L 2008a: Acta Geod. Geoph. Hung. (present issue)

Szalai S, Szarka L 2008b: Geophys. Prosp., doi:10.1111/j.1365-2478.2007.00673.x, 56, $159-175$.

Szarka L 1994: Possibilities of three-dimensional geological structures by using electromagnetic geophysical methods. Thesis for the degree of Doctor of Science. Hungarian Academy of Sciences, Budapest

Zhdanov M S, Keller G 1994: The geoelectrical methods in geophysical exploration. Elsevier, Amsterdam-New York-Tokyo 